\title{
Awake Nonresectional Lung Volume Reduction Surgery
}

\author{
Tommaso Claudio Mineo, MD, Eugenio Pompeo, MD, Davide Mineo, MD, Frederico Tacconi, MD, \\ Mario Marino, MD, and Alessandro Fabrizio Sabato, MD
}

\begin{abstract}
Objective: To assess the feasibility, safety, and early results of awake lung volume reduction surgery (LVRS) performed under thoracic epidural anesthesia by a new nonresectional technique.

Summary Background Data: So far, resectional LVRS under general anesthesia and one-lung ventilation is the more frequently used technique, but procedure-related morbidity has been considerable. Methods: The study cohort included 12 patients undergoing unilateral awake LVRS. Evaluated parameters included technical feasibility and anesthesia satisfaction scored into 4 grades (from $1=$ poor to $4=$ excellent), global operating room time, and arterial carbon dioxide tension $\left(\mathrm{PaCO}_{2}\right)$. In addition, 6-month changes in outcome measures, including forced expiratory volume in 1 second (FEV1), residual volume (RV), 6-minute walking test (SMWT), and dyspnea index were recorded. Perioperative and 6-month results were comparable with those of a control group undergoing unilateral resectional LVRS.
\end{abstract}

Results: Technical feasibility was excellent to satisfactory in 11 patients. One patient required conversion to one-lung ventilation. Differences between the awake and control group included global operating room time $(90 \pm 17$ minutes versus $145 \pm 19$ minutes, $P<0.00001)$; $\mathrm{PaCO}_{2} 24$ hours after surgery (45 $\pm 6 \mathrm{~mm} \mathrm{Hg}$ versus $49 \pm 6 \mathrm{~mm} \mathrm{Hg}, P=0.02)$; and hospital stay $(7.8 \pm 5$ days versus $11.7 \pm 4$ days, $P=0.02)$. Significant $(P<0.002)$ improvements occurred at 6 months in FEV1 $(0.31 \pm 0.17 \mathrm{~L}), \mathrm{RV}(-1.41 \pm 0.7$ $\mathrm{L})$, SMWT $(73 \pm 25 \mathrm{~m})$, and dyspnea index $(-1.3 \pm 0.5)$ and were comparable with those of the control group.

Conclusions: In this study, awake nonresectional LVRS proved feasible and safe. This new modality was associated with a faster recovery and satisfactory 6-month outcome, which did not differ from that of resectional LVRS.

(Ann Surg 2006;243: 131-136)

From the Division of Thoracic Surgery, Multidisciplinary Pulmonary Program, Tor Vergata School of Medicine, Policlinico Tor Vergata, Rome, Italy.

Supported by MURST COFIN Grant Nos. 9906274194-06 and No. 2001061191-001, CNR No. CU0100935 2002, and Centro di Eccellenza 2001. This study was carried out within the Research Fellowship Program Tecnologie e Terapie Avanzate in Chirurgia awarded by the Tor Vergata University.

Reprints: Eugenio Pompeo, MD, Cattedra di Chirurgia Toracica, Università Tor Vergata, Policlinico Tor Vergata, V.le Oxford, 81, 00133 Rome, Italy. E-mail: pompeo@med.uniroma2.it.

Copyright (C) 2005 by Lippincott Williams \& Wilkins

ISSN: 0003-4932/06/24301-0131

DOI: $10.1097 / 01$. sla.0000182917.39534.2c ung volume reduction surgery (LVRS) can offer sustained Limprovements in respiratory function, exercise capacity, subjective dyspnea, and quality of life in properly selected patients with severe emphysema. ${ }^{1-4}$ The most widely used surgical technique entails unilateral or bilateral staple resection of the most emphysematous lung tissue..$^{1-4}$ It is performed under general anesthesia with single-lung ventilation through open or thoracoscopic approaches. Unfortunately, definitive acceptance of LVRS is still hampered by a certain operative mortality and a considerable morbidity, which represent major shortcomings of the procedure and lead to large economic costs. ${ }^{5}$ The main causes of morbidity and mortality can be addressed to general anesthesia, surgical trauma, and complications arising from resection of emphysematous lung tissue, most notably prolonged air leaks. ${ }^{1-4}$

We have hypothesized that awake LVRS under thoracic epidural anesthesia (TEA) might be feasible and could be associated with a less morbid and more rapid recovery due to the avoidance of general anesthesia-related adverse effects. ${ }^{6}$ We also reasoned that a thoracoscopic nonresectional LVRS technique entailing introflexing plication of the most emphysematous lung regions could optimally apply to awake LVRS and might offer some advantages compared with the resectional technique.

We have thus undertaken a pilot study to assess feasibility, safety, and early results of awake thoracoscopic LVRS performed by this new technique.

\section{MATERIALS AND METHODS}

The study comprised 12 patients who underwent awake intentional unilateral LVRS surgery between January 2001 and January 2002. Informed consent was obtained from all patients once they were informed about the investigational nature of the study, the risks and potential benefit of the procedure, as well as the possibility that conversion to general anesthesia and single-lung ventilation could be necessary in case of unexpected technical or anesthesiologic problems. The study was approved by the ethical committee of our University. Primary outcome measures were technical feasibility and anesthesia satisfaction scored into 4 grades $(1=$ unsatisfactory; 2 = satisfactory; $3=$ good; $4=$ excellent), hospital stay and 6-month changes in forced expiratory volume in 1 second $\left(\mathrm{FEV}_{1}\right)$, residual volume (RV), 6 minute walking test (SMWT), maximal incremental treadmill test (MITT), modified Medical Research Council dyspnea index score, and short form-36 item quality of life (SF-36) physical functioning $(\mathrm{PF})$ domain score. 
Secondary outcome measures were anesthesia time, operative time, global in-operating room time, and operative mortality and morbidity. In addition, the ratio of arterial oxygen tension to fraction of inspired oxygen $\left(\mathrm{PaO}_{2} / \mathrm{FiO}_{2}\right)$ and arterial carbon dioxide tension $\left(\mathrm{PaCO}_{2}\right)$ was assessed preoperatively, intraoperatively at the completion of the LVRS procedure, and 24 hours after surgery.

The study was conducted following a 2-step plan. The first step included an initial subset of 6 patients operated on. To proceed with the second 6-patient-based subset, it was assumed that in the first cohort the procedure could be safely completed through sole TEA in at least 4 patients with no major side effects, and an operative time, morbidity rate, and hospital stay, which did not negatively differ at an interim analysis from those observed in an historical cohort treated by resectional LVRS under general anesthesia.

Inclusion criteria were those applied for standard LVRS $^{3}$ and included severe disability despite maximized medical care, postbronchodilator $\mathrm{FEV}_{1}$ less than $40 \%$ predicted, RV more than $180 \%$ predicted, and the finding of upper lobe prevailing, heterogeneous emphysema at radiologic workup. In addition, to be included in the study, patients should have radiologic evidence of distinct heterogeneity of emphysema between the lungs (ie, asymmetric emphysema), which at our institution is an indication for intentional unilateral LVRS.

Contraindications for awake LVRS regarded radiologic evidence of extensive pleural adhesions with pleural scarring and calcifications.

Contraindications for TEA included patient's refusal or noncompliance, unfavorable anatomy, previous surgery of the cervical or upper thoracic spine, compromised coagulation (thromboplastin time $<80 \%$, prothrombin time $>40 \mathrm{~s}$, or platelets $<100 / \mathrm{nL}$ ) or bleeding disorder.

\section{Physiologic Assessment}

Static lung volumes were determined by plethysmography, and diffusing capacity for carbon monoxide was assessed by the single-breath technique. Pulmonary function tests were performed after administration of 2 puffs of aerosolized salbutamol. Reference spirometric values were those of the European Respiratory Society. ${ }^{7}$ Exercise tolerance was assessed by standard SMWT and MITT. Dyspnea was rated according to the modified Medical Research Council Score. All baseline tests were performed during periods of clinical stability and within 1 week after completion of the preoperative rehabilitation program.

Criteria for discharge were standardized and included stable clinical conditions with oxygen saturation at rest $\geq 90 \%$ and all chest tubes removed. Removal of chest tubes was performed after that a chest roentgenogram demonstrated complete reexpansion of the lung and 24 hours after any air leak stopped, and drainage fluid did not exceed $150 \mathrm{~mL}$.

All primary and secondary outcome measures, excepted technical feasibility and anesthesia satisfaction scores, were compared with those of a historical control group constituted by the last 12 patients undergoing intentional unilateral resectional LVRS at our institution.

\section{Anesthesia}

The objective of TEA was to achieve somatosensory and motor block at the T1 to T8 level. One of the major objectives was to achieve motor block of the intercostal muscles while preserving diaphragmatic respiration. The thoracic epidural catheter was inserted at $\mathrm{T} 4$ after patients were premedicated orally with $7.5 \mathrm{mg}$ midazolam. In the operating room, patients received a continuous infusion of ropivacaine $0.5 \%$ and sufentanil $1.66 \mu \mathrm{g} / \mathrm{mL}$ into the epidural space. During the procedure, patients breathed $\mathrm{O}_{2}$ through a venturi mask/face to keep oxygen saturation above 90\%. During wound closure, the anesthetic regimen was changed to ropivacaine $0.16 \%$ and sufentanil $1 \mu \mathrm{g} / \mathrm{mL}$ at 2 to $5 \mathrm{~mL} / \mathrm{hr}$. All patients received lactated Ringer's solution at $12 \mathrm{~mL} \cdot \mathrm{kg}^{-1} \cdot \mathrm{h}^{-1}$.

Patients undergoing resectional LVRS through general anesthesia had received a thoracic epidural catheter inserted between T5 and T8 and a continuous infusion of ropivacaine. General anesthesia was induced with intravenous propofol $(1.5-2 \mathrm{mg} / \mathrm{kg})$, fentanyl $(0.1 \mathrm{mg})$ and vecuronium $(0.1 \mathrm{mg} /$ $\mathrm{kg}$ ) and maintained using a continuous infusion of propofol, fentanyl, and vecuronium. A left-sided double-lumen tube was routinely used. In all patients, epidural catheter was removed 48 hours after surgery.

\section{Surgical Technique}

The patient was placed in full lateral decubitus position. Four flexible thoracoscopic trocars were inserted. The camera port was placed in the sixth intercostal space along the midaxillary line while operating ports were placed in the third and fifth intercostal space along the anterior axillary line, and in the fourth intercostal space along the posterior axillary line. A $30^{\circ}, 10-\mathrm{mm}$ camera was used to facilitate oblique vision of the lung during spontaneous ventilation. The most emphysematous target areas were visualized and introflexed with a cotton swab while redundant lung edges were gently grasped by 2 ring forceps (Fig. 1A). Subsequently, both lung edges were grasped together with a single ring forceps and a

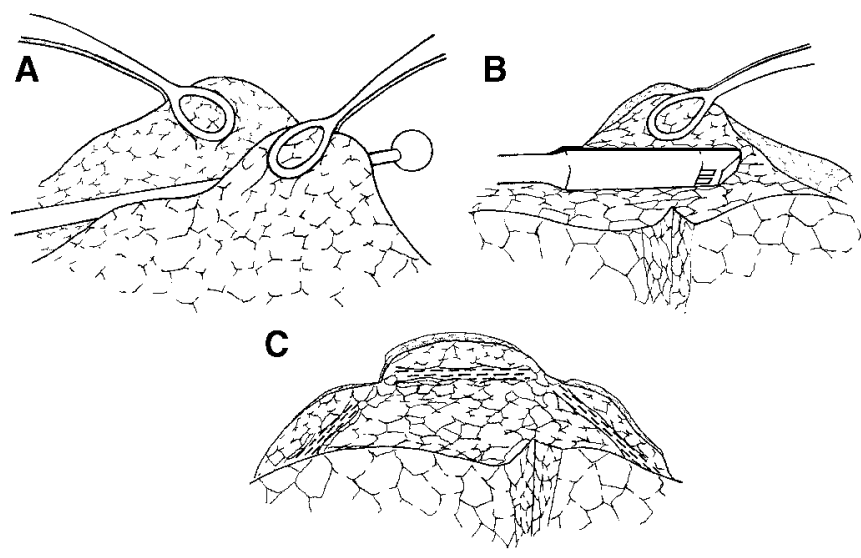

FIGURE 1. Edges of the most emphysematous regions are grasped by 2 ring forceps while lung tissue is introflexed by a cotton swab (A); endoscopic "no knife" stapler is applied at the periphery of the plicated area (B); at the completion of the procedure, 3 staples are fired so that the upper lobe lung volume is reduced by about $50 \%(\mathrm{C})$. 

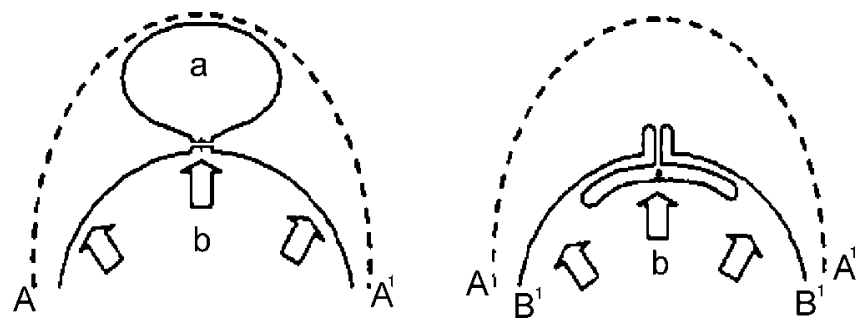

FIGURE 2. Schematic sagittal drawing illustrating the conceptual difference between resectional LVRS (left) and introflexing lung plication (right). In resectional LVRS, the overall lung volume $\left(A-A^{1}\right)$ is reduced $(b)$ through resection of the most emphysematous lung region (a). The resulting suture line is continuous and placed deep into the lung lobe, thus involving subsegmental bronchi and vessels. Reexpansion forces (central arrow) are exerted directly on the suture line. In introflexing lung plication, equivalent loss of lung volume is achieved without resection of lung tissue; the staple suture line is interrupted and peripheral so that vessels and bronchi are less likely to be involved; furthermore, lung reexpansion forces are not directly applied on the suture due to the "inlay" buttress of the plicated visceral pleura $\left(B-B^{1}\right)$ itself.

45-mm, "no knife" endostapler with 3.5-mm cartridges was applied on the plicated lung region starting at the apex of the upper lung lobe (Fig. 1B). In a similar manner, 2 other cartridges were fired in the ventral and dorsal side of the targeted area to perform a linear, interrupted suture line (Fig. 1C). As a result, the upper lobe volume was reduced by about $50 \%$ without any loss of lung tissue (Fig. 2). At the end of the procedure, 2 chest tubes were placed in the pleural cavity.

In the control group, staple resection of target areas was performed excising a reversed U-shaped single strip of emphysematous lung tissue to reduce the upper lobe of about $50 \%$. No suture line buttress was used in either group.

After the completion of the operation, patients undergoing awake LVRS were immediately transferred to the ward and started drinking and walking.

In the control group, drinking, eating, and walking were routinely resumed on postoperative day 1 . All unstable patients $\left(\mathrm{SaO}_{2}<90 \%\right.$ with $\mathrm{FiO}_{2}$ at $35 \%$ and $/$ or $\mathrm{PaCO}_{2}>45 \mathrm{~mm}$ $\mathrm{Hg}$ ) stayed in the postoperative intensive care unit for at least 24 hours.

\section{Statistics}

Group descriptive statistics are presented as mean \pm SD. Because of the small sample size, the nonparametric Wilcoxon rank sum test and the Mann-Whitney $U$ test were used for paired and unpaired data, respectively. Frequencies were compared with a 2-tailed Fisher exact test. A $P$ value of less than 0.05 was considered significant.

\section{RESULTS}

Comparison of demographics and baseline data indicates that the groups were relatively well matched (Table 1).

One patient in the awake LVRS group required conversion to general anesthesia and one-lung ventilation due to the presence of unexpected diffuse pleural adhesions. Tech-
TABLE 1. Demographics and Preoperative Data in the Study Groups

\begin{tabular}{lccc}
\hline & $\begin{array}{c}\text { Awake Group } \\
(\mathbf{n}=\mathbf{1 2})\end{array}$ & $\begin{array}{c}\text { Control Group } \\
(\mathbf{n}=\mathbf{1 2})\end{array}$ & $\begin{array}{c}\text { Intergroup } \\
\boldsymbol{P} \text { Value }\end{array}$ \\
\hline Age (yr) & $64 \pm 6$ & $65 \pm 8$ & 0.9 \\
Sex ratio (F:M) & $1: 11$ & $0: 12$ & 0.9 \\
Severity of & $26 \pm 6$ & $28 \pm 7$ & 0.5 \\
$\quad$ emphysema (score) & $1.3 \pm 0.1$ & $1.2 \pm 0.1$ & 0.1 \\
Asymmetric ratio of & & & \\
$\quad$ emphysema (score) & $1.0 \pm 0.4$ & $0.9 \pm 0.2$ & 0.9 \\
FEV1 (L) & $35 \pm 14$ & $33 \pm 9$ & 0.9 \\
FEV1 (\%) & $2.5 \pm 0.7$ & $2.5 \pm 0.5$ & 0.5 \\
FVC (L) & $69 \pm 14$ & $68 \pm 13$ & 0.9 \\
FVC (\%) & $5.1 \pm 0.7$ & $4.9 \pm 0.5$ & 0.4 \\
RV (L) & $218 \pm 40$ & $210 \pm 26$ & 0.7 \\
RV (\%) & $2.9 \pm 0.7$ & $2.7 \pm 0.5$ & 0.4 \\
Dyspnea index & & & \\
$\quad$ (score) & $72 \pm 8$ & $68 \pm 7$ & 0.2 \\
PaO (mm Hg) & $39 \pm 2$ & $41 \pm 3$ & 0.01 \\
PaCO (mm Hg) & $38 \pm 9$ & $38 \pm 8$ & 0.8 \\
$\left.\mathrm{DLCO}_{2} \%\right)$ & $399 \pm 55$ & $375 \pm 63$ & 0.3 \\
$\mathrm{SMWT}_{\text {(m) }}$ & $0.9 \pm 0.4$ & $0.9 \pm 0.7$ & 0.6 \\
$\mathrm{MITT} \mathrm{(Bruce} \mathrm{class)}_{\mathrm{PF} \text { (score) }}$ & $22 \pm 18$ & $20 \pm 16$ & 0.8 \\
\hline
\end{tabular}

FEV1 indicates forced expiratory volume in 1 second; FVC, forced vital capacity; $\mathrm{RV}$, residual volume; $\mathrm{PaO}_{2}$, arterial oxygen tension; $\mathrm{PaCO}_{2}$, arterial carbon dioxide tension; DLCO, diffusion of carbon monoxide; SMWT, 6-minute walking test; MITT, maximal incremental treadmill test; PF, SF-36 physical functioning domain score; NS, not significant.

nical feasibility was scored by the surgeon as excellent in 7 patients, good in 3 patients, and satisfactory in 1 . No patient required conversion to thoracotomy.

Comparative analysis of perioperative data showed that awake LVRS resulted in shorter anesthesia time, operative time, and global operating room time. Intraoperatively, there was no significant difference in $\mathrm{PaO}_{2} / \mathrm{FiO}_{2}$, whereas $\mathrm{PaCO}_{2}$ was higher in the awake group. On the other hand, 24 hours after surgery, $\mathrm{PaCO}_{2}$ was significantly higher in the control group (Table 2).

As a whole, awake LVRS was well tolerated by the patients, and satisfaction with the type of anesthesia was scored as excellent by 7 patients, as good by 4 patients, and as satisfactory by 1 patient. Postoperative intensive care unit stay was necessary in 9 patients in the control group and none in the awake group. In the awake group, all patients were allowed to drink, eat, and walk as desired within a few hours after the operation. In the control group, 10 patients could start drinking, eating, and walking on postoperative day 1, while 2 patients could start drinking, eating, and walking on postoperative day 2 and 3, respectively. There was no difference between the groups in need for combined analgesia at the fourth postoperative day. No patient complained of nausea postoperatively, while 1 patient in either group had urinary retention, which required temporary urinary catheterization.

Ninety-day mortality was nil in both groups. The most relevant nonfatal complication regarded prolonged air leaks ( $>7$ days), which occurred in 3 patients in the awake group 
TABLE 2. Perioperative Data in the Study Groups

\begin{tabular}{|c|c|c|c|}
\hline & $\begin{array}{l}\text { Awake Group } \\
\quad(\mathrm{n}=12)\end{array}$ & $\begin{array}{l}\text { Control Group } \\
(\mathrm{n}=12)\end{array}$ & $\begin{array}{c}\text { Intergroup } \\
P \text { Value }\end{array}$ \\
\hline Anesthesia time (min) & $40 \pm 6$ & $48 \pm 12$ & 0.02 \\
\hline Surgery time (min) & $48 \pm 12$ & $68 \pm 13$ & 0.001 \\
\hline Weaning time (min) & - & $28 \pm 9$ & - \\
\hline $\begin{array}{l}\text { Global operating room } \\
\text { time (min) }\end{array}$ & $88 \pm 12$ & $145 \pm 19$ & $<0.00001$ \\
\hline $\begin{array}{l}\text { Intensive care unit stay } \\
\text { (hr) }\end{array}$ & - & $29 \pm 12$ & - \\
\hline $\begin{array}{l}\text { Technical feasibility } \\
\text { (score) }\end{array}$ & $3.3 \pm 1$ & - & - \\
\hline $\begin{array}{l}\text { Anesthesia satisfaction } \\
\text { (score) }\end{array}$ & $3.5 \pm 0.7$ & - & - \\
\hline Intraoperative $\mathrm{PO}_{2} / \mathrm{FiO}_{2}$ & $191 \pm 61$ & $178 \pm 23$ & 0.6 \\
\hline $\begin{array}{l}\text { Intraoperative } \mathrm{PCO}_{2} \\
\quad(\mathrm{~mm} \mathrm{Hg})\end{array}$ & $57 \pm 9$ & $41 \pm 3$ & $<0.00001$ \\
\hline Postoperative $\mathrm{PO}_{2} / \mathrm{FiO}_{2}$ & $158 \pm 23$ & $149 \pm 28$ & 0.5 \\
\hline $\begin{array}{l}\text { Postoperative } \mathrm{PCO}_{2} \\
\quad(\mathrm{~mm} \mathrm{Hg})\end{array}$ & $46 \pm 6$ & $49 \pm 6$ & 0.02 \\
\hline Mortality (N) & 0 & 0 & - \\
\hline Morbidity $(\mathrm{N})$ & 3 & 6 & 0.4 \\
\hline Hospital stay (days) & $7.8 \pm 5$ & $11.7 \pm 4$ & 0.02 \\
\hline
\end{tabular}

Postoperative assessment was performed on postoperative day 1 .

versus 6 patients in the control group. In accordance with this finding, hospital stay was significantly shorter in the awake group (Table 2). In particular, in the awake group, 6 patients had a hospital stay of 5 days or less while in the control group no patient stayed in the hospital less than 7 days. No patient in either group was discharged with a Heimlich valve.

After 6 months, 1 patient in the control group had died due to respiratory failure. Significant preoperative to postoperative improvements occurred in both groups in $\mathrm{FEV}_{1}$, forced vital capacity, RV, 6MWT, MITT, dyspnea index, and SF-36 PF domain score with no significant differences at intergroup comparison (Table 3).

TABLE 3. Six Months Absolute Changes in Clinical Outcome Measures

\begin{tabular}{lrrc}
\hline & Awake Group & Control Group & $\begin{array}{c}\text { Intergroup } \\
\boldsymbol{P} \text { Value }\end{array}$ \\
\hline$\Delta$ FEV1 (L) & $0.31 \pm 0.17^{*}$ & $0.22 \pm 0.14^{*}$ & 0.3 \\
$\Delta$ FVC (L) & $0.47 \pm 0.3^{*}$ & $0.33 \pm 0.2^{*}$ & 0.2 \\
$\Delta \mathrm{RV}(\mathrm{L})$ & $-1.41 \pm 0.7^{*}$ & $-0.96 \pm 0.4^{*}$ & 0.1 \\
$\Delta$ SMWT (m) & $73 \pm 25^{*}$ & $59 \pm 33^{*}$ & 0.2 \\
$\Delta$ MITT (Bruce class) $^{*}$ & $1.1 \pm 0.3^{*}$ & $0.84 \pm 0.6^{\dagger}$ & 0.3 \\
$\Delta \mathrm{PO}_{2}$ (mm Hg) & $2.6 \pm 3.4^{\dagger}$ & $0.4 \pm 1.7$ & 0.1 \\
$\Delta$ dyspnea index (score) & $-1.3 \pm 0.5^{*}$ & $-0.8 \pm 0.7^{\dagger}$ & 0.1 \\
$\Delta$ PF (SF-36 score) & $32 \pm 9^{*}$ & $25 \pm 13^{\dagger}$ & 0.1 \\
\hline
\end{tabular}

Within-group preoperative to postoperative changes: ${ }^{*} P<0.005 ;{ }^{\dagger} P<0.03$.

FEV1 indicates forced expiratory volume in 1 second; FVC, forced vital capacity; $\mathrm{RV}$, residual volume; SMWT, 6-minute walking test; MITT, maximal incremental treadmill test; $\mathrm{PO}_{2}$, arterial oxygen tension; $\mathrm{PF}, \mathrm{SF}-36$ physical functioning domain score.

\section{DISCUSSION}

One-lung ventilation under general anesthesia is deemed necessary for most thoracic surgery procedures. However, several adverse effects can derive from this type of anesthesia, including an increased risk of pneumonia, impaired cardiac performance, and neuromuscular problems. Moreover, mechanical ventilation can cause damage, which includes barotrauma, volotrauma, and atelectrauma. ${ }^{6}$ Yet, it has been found that atelectasis in the dependent lung is a common finding during general anesthesia with muscle paralysis. In these instances, a right-to-left shunt of blood can develop and correlate with the size of atelectasis. ${ }^{7,8}$ Moreover, prolonged collapse of the nondependent lung during one-lung ventilation can delay its complete reexpansion after weaning, thus leading to the development of further atelectasic areas. Theoretically, avoidance of general anesthesia and one-lung mechanical ventilation might offer several advantages, particularly in functionally compromised emphysematous patients.

The major finding of this study is that awake nonresectional LVRS was safely and easily performed under TEA. This result seems to contradict the accepted assumption that the main prerequisite for allowing lung surgery is general anesthesia with one-lung ventilation. Furthermore, introflexing lung plication revealed an ideal awake LVRS technique because of its easy feasibility and safety.

Awake nonresectional LVRS was readily accepted and well tolerated by the patients. Avoidance of general anesthesia reflected in satisfactory perioperative respiratory performance and allowed a more rapid recovery and immediate return to many daily life activities, including drinking, eating, and walking. In addition, 90-day mortality was nil in either group, whereas nonfatal complications were less frequent in the awake group. As a result, hospital stay was significantly shorter than that of the control group. Finally, at the 6-month follow-up, significant incremental improvements occurred in clinical and respiratory function measures after awake nonresectional LVRS, and these improvements did not differ from those achieved in the control group.

One of our major concerns was that operating on a ventilated lung would have rendered surgical maneuvers more difficult because of the lung movements and the limited space for surgical maneuvering. Instead, we have immediately noticed that the open pneumothorax created during the operation determined sufficient lung collapse, which could even be progressively increased by gentle instrumental lung compression.

Theoretically, an open pneumothorax could have compressed the dependent lung, eventually resulting in functional compromise. However, apart from a temporary permissive hypercapnia, respiratory function remained satisfactory throughout the procedure. We hypothesize that maintained diaphragmatic motion could have decreased the detrimental effect of the abdominal pressure, leading the paralyzed diaphragm to compress the dependent lung during general anesthesia. $^{9}$

In this study, the perioperative rise in $\mathrm{PaCO}_{2}$ was well tolerated by the patients and resolved more rapidly than in the control group. We hypothesize that this may be due to better 
synchronization of rib cage-abdominal motion, which was immediately observed after awake nonresectional LVRS. Indeed, the greater contribution of abdominal volume changes to tidal volume is consistent with a reduction of inspiratory loading and a greater force-generating capacity of the diaphragm due to resizing of the chest and diaphragmatic dome after LVRS. ${ }^{10}$ Unfortunately, these beneficial effects of LVRS are lost in the early postoperative period in patients receiving general anesthesia.

Lung plication techniques for surgical treatment of severe emphysema have been already described by others surgeons and have recently demonstrated to allow satisfactory clinical results and acceptable morbidity. ${ }^{11-13}$ However, all the proposed techniques have been performed under general anesthesia and entail suturing at the base of the bullous region, which does not prevent the typical adverse effects of resectional LVRS.

We have observed that the introflexing lung plication technique, although following the same basic concepts of resectional LVRS including a reduction of $20 \%$ to $30 \%$ of the lung volume, a suture line applied along a single ideal line, and the use of stapling devices, proved simpler and quicker than resectional LVRS and might offer some advantages. Indeed, a peripheral suture line does not involve either interruption of subsegmental bronchi and vessels or resection of healthy tissue. Moreover, a linear but interrupted suture line is more flexible than a continuous one, theoretically reducing the risk of tears on the lung surface during lung reexpansion. Finally, introflexing lung plication might offer a more uniform distribution of the lung expansion forces around the suture line due to the inlay buttress created by the plicated bullous tissue itself. All these theoretical advantages, although not demonstrable by this pilot study, might have contributed to determine the reduced incidence of prolonged air leaks and the shorter hospitalization we have observed in the awake group. Moreover, postoperative radiologic study demonstrated that following awake nonresectional LVRS, satisfactory reconfiguration of diaphragmatic dome and rib cage did occur. Yet, high-resolution CT scan showed that there was no visible suture line within the lung tissue due to the peripheral location of the staple sutures (Fig. 3).

The rapidity of the procedure together with the significantly reduced anesthesia time and the lack of weaning time resulted in a significantly shorter in-operating room time, which offered the double advantage of less risk for the patient and less cost for the institution.

Awake major surgery under TEA has been recently reported and included aortic aneurysm repair in patients with severe pulmonary disease ${ }^{14}$ and coronary artery bypass grafting. ${ }^{15,16}$ Furthermore, TEA has been successfully used for thoracoscopic management of secondary pneumothorax ${ }^{17}$ and more recently for resection of solitary pulmonary nodules. ${ }^{18}$ The promising results of such initial experiences encouraged us to perform awake nonresectional LVRS. Nonetheless, TEA has potential complications, including epidural hematoma and spinal cord injury. A further potential risk of high TEA is phrenic nerve palsy caused by inadvertently high anesthetic level. On the other hand, with awake nonresectional LVRS, the risk of postopera-
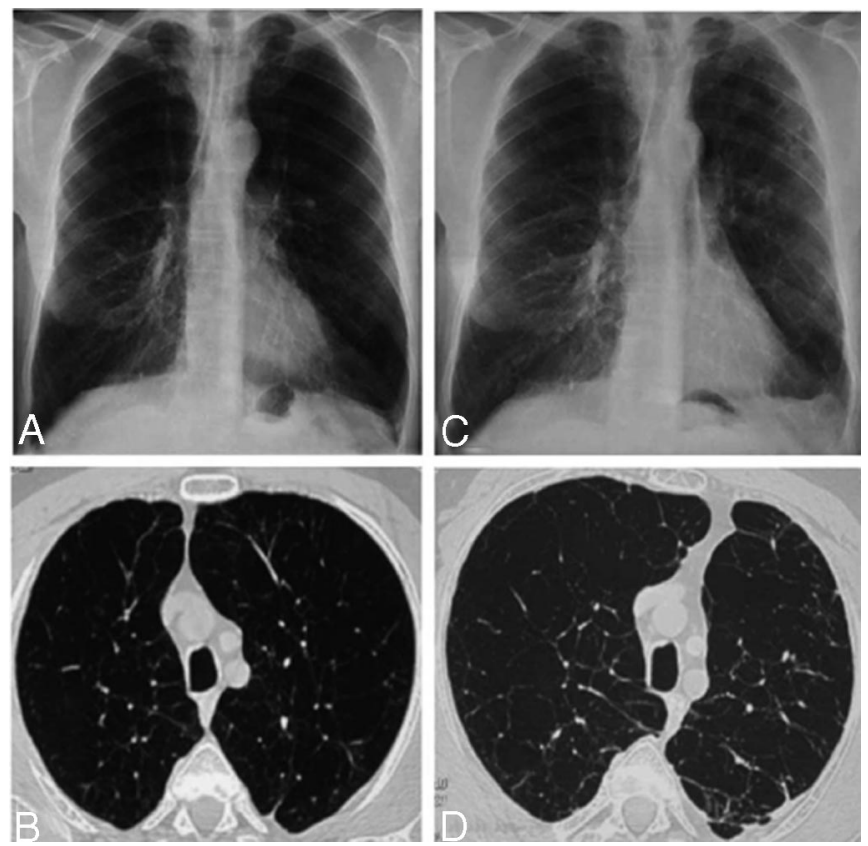

FIGURE 3. Preoperative (A, B) and postoperative (C, D) radiologic study of a patient undergoing awake nonresectional LVRS. Postoperative chest roentgenogram (C, D) and high resolution computed tomography (D) show the meaningful reduction of the left lung volume and the absence of suture line within the lung tissue due to the peripheral suturing achieved through introflexing lung plication.

tive pulmonary failure and long-term ventilation may be reduced and many potential risks of endotracheal intubation, including trauma to teeth or vocal cords and peri-intubational hypoxia, are avoided.

We thus believe that this new surgical modality is a promising adjunct to current minimally invasive LVRS techniques and facilitated the direct transfer of the patient from the operating room to the ward. Such policy, which is part of a new strategy, namely, fast-track pathway, is being currently applied in cardiac surgery and should be pursued in thoracic surgery as well.

We acknowledge some limitations of our study. First, the small study population and the limited follow-up did not allow us to draw definitive conclusions on the efficacy of awake nonresectional LVRS. Second, the sequential and not simultaneous intergroup comparison of this study makes possible the risk that the more recent series (ie, the awake group) could have taken advantage from tricks and tips learned with the former group. However, the 24 patients included in our analysis embrace a very limited period of time within our LVRS program, which started in 1995, a finding that renders such risk negligible. Finally, it is our opinion that until more experience will be gained, this awake technique applies optimally for unilateral treatment; and to be used in patients requiring bilateral treatment, it should be better performed following a staged approach.

Our surgical technique and strategy in LVRS are evolving over time. We previously thought that a thoracoscopic 
approach might be beneficial in delicate emphysematous patients; we also advocated a unilateral approach either alone or as the first step within a staged bilateral strategy in properly selected patients. ${ }^{19}$ More recently, we have performed awake LVRS under sole TEA to further reduce the invasiveness of the procedure. With this new approach, we have now investigated the feasibility, safety, and early results of a nonresectional LVRS technique entailing introflexing lung plication, which might reduce the procedure-related morbidity without jeopardizing the clinical benefit demonstrated with resectional LVRS. Although a prospective randomized study is warranted to eventually confirm or contradict our findings, we keep in mind the provocative sentence reported by Fessler and $\mathrm{Wise}^{20}$ to define the rationale of LVRS, which states "less is more." Indeed, the future of LVRS might be strictly related to the satisfaction of this intriguing philosophical assumption.

\section{REFERENCES}

1. National Emphysema Treatment Trial Research Group. A randomized trial comparing lung-volume-reduction surgery with medical therapy for severe emphysema. N Engl J Med. 2003;348:2059-2073.

2. Goldstein RS, Todd TRJ, Guyatt G. Influence of lung volume reduction surgery (LVRS) on health related quality of life in patients with chronic obstructive pulmonary disease. Thorax. 2003;58:405-410.

3. Pompeo E, Marino M, Nofroni I, et al. Reduction pneumoplasty versus respiratory rehabilitation: a randomised trial. Ann Thorac Surg. 2000; 70:948-954.

4. Ciccone AM, Meyers BF, Guthrie TJ, et al. Long-term outcome of bilateral lung volume reduction in 250 consecutive patients with emphysema. J Thorac Cardiovasc Surg. 2003;125:513-525.

5. National Emphysema Treatment Trial Research group. Cost effectiveness of lung-volume-reduction surgery for patients with severe emphysema. N Engl J Med. 2003;348:2092-2102.

6. Whitehead T, Slutsky AS. The pulmonary physician in critical care: ventilator induced lung injury. Thorax. 2002;57:635-642.
7. Quanjer PH, Tamelling GJ, Cotes JE, et al. Lung volumes and forced expiratory flows: report working party standardization of lung function tests, European Community for Steel and Coal. Official Statement of European Respiratory Society. Eur Respir J. 1993;6(suppl 16):5-40.

8. Tokics L, Hedenstierna G, Svensson L, et al. V/Q distribution and correlation to atelectasis in anesthetized paralyzed humans. $J$ Appl Physiol. 1996;81:1822-1833.

9. Cohen E. Physiology of the lateral position and one-lung ventilation. Chest Surg Clin North Am. 1997;7:753-771.

10. Bloch KE, Li Y, Zhang J, et al. Effect of surgical lung volume reduction on breathing patterns in severe pulmonary emphysema. Am J Respir Crit Care Med. 1997;156:553-560.

11. Crosa-Dorado VL, Pomi J, Perez-Penco EJ, et al. Treatment of dyspnea in emphysema: pulmonary remodeling: hemo- and pneumostatic suturing of the emphysematous lung. Res Surg. 1992;4:1-4.

12. Swanson SJ, Mentzer SJ, DeCamp M Jr, et al. No-cut thoracoscopic lung plication: a new technique for lung volume reduction surgery. $J$ Am Coll Surg. 1997;185:25-32.

13. Iwasaki M, Nishiumi N, Kaga K, et al. Application of the fold plication method for unilateral lung volume reduction in pulmonary emphysema. Ann Thorac Surg. 1999;67:815-817.

14. McGregor WE, Koler AJ, Labat GC, et al. Awake aortic aneurysm repair in patients with severe pulmonary disease. Am J Surg. 1999;178:121124.

15. Kessler P, Neidhart G, Bremerich DH, et al. High thoracic epidural anesthesia for coronary artery bypass grafting using two different surgical approaches in conscious patients. Anesth Analg. 2002;95:791-797.

16. Aybek T, Kessler P, Dogan S, et al. Awake coronary artery bypass grafting: utopia or reality? Ann Thorac Surg. 2003;75:1165-1170.

17. Mukaida T, Andou A, Aoe M, et al. Thoracoscopic operation for secondary pneumothorax under local and epidural anesthesia in highrisk patients. Ann Thorac Surg. 1998;65:924-926.

18. Pompeo E, Mineo D, Rogliani P, et al. Feasibility and results of awake thoracoscopic resection of solitary pulmonary nodules. Ann Thorac Surg. 2004;78:1761-1768.

19. Mineo TC, Pompeo E, Mineo D, et al. Results of unilateral lung volume reduction surgery in patients with distinct heterogeneity of emphysema between lungs. J Thorac Cardiovasc Surg. 2005;129:73-79.

20. Fessler HE, Wise RA. Lung volume reduction surgery: is less really more? Am J Respir Crit Care Med. 1999;159:1031-1035. 